Meta

Journal des traducteurs

Translators' Journal

\title{
Les corpus publicitaires : nouvelles approches et méthodes pour le traducteur
}

\section{Mathieu Guidère}

Volume 56, numéro 2, juin 2011

Les corpus et la recherche en terminologie et en traductologie Corpora and Research in Terminology and Translation Studies

URI : https://id.erudit.org/iderudit/1006180ar

DOI : https://doi.org/10.7202/1006180ar

Aller au sommaire du numéro

Éditeur(s)

Les Presses de l’Université de Montréal

ISSN

0026-0452 (imprimé)

1492-1421 (numérique)

Découvrir la revue

Citer cet article

Guidère, M. (2011). Les corpus publicitaires : nouvelles approches et méthodes pour le traducteur. Meta, 56(2), 336-350. https://doi.org/10.7202/1006180ar

\section{Résumé de l'article}

Il y a quelques années, la constitution et l'exploitation des corpus publicitaires n'étaient pas une tâche aisée, mais la généralisation de l'internet comme outil de communication sociale, commerciale et institutionnelle a révolutionné ce champ d'études en rendant accessibles des sources d'information riches et variées. Les messages publicitaires sont désormais accessibles en ligne et en plusieurs langues, sur un même site web. Ils forment un corpus virtuel, multilingue et intersémiotique, offrant des textes parallèles et simultanés. Cette accessibilité autorise le traducteur à envisager de nouvelles approches et méthodes d'analyse. Tout d'abord, pour la constitution même des corpus d'étude, l'internet offre aujourd'hui la possibilité de réunir en un temps record des corpus multilingues et multimédias sans équivalent. Ensuite, pour leur exploitation, la comparaison des éléments constitutifs de ces corpus offre de nouvelles méthodes de recherche qui permettent d'envisager le traducteur comme un véritable communicateur. Enfin, en ce qui concerne la conceptualisation, une approche interdisciplinaire de ces corpus ouvre des perspectives inédites en matière d'études traductologiques et communicationnelles. Nous aborderons ces différents niveaux d'analyse en partant d'études de cas réalisées à une décennie d'intervalle à partir de corpus publicitaires multilingues. Notre objectif est d'expliquer l'évolution qui s'est opérée ces dernières années dans le domaine de la traduction publicitaire et de montrer l'intérêt d'une approche diachronique des corpus pour la traductologie.
Ce document est protégé par la loi sur le droit d'auteur. L'utilisation des services d'Érudit (y compris la reproduction) est assujettie à sa politique d'utilisation que vous pouvez consulter en ligne.

https://apropos.erudit.org/fr/usagers/politique-dutilisation/ 


\title{
Les corpus publicitaires: nouvelles approches et méthodes pour le traducteur
}

\author{
MATHIEU GUIDÈRE \\ Université de Toulouse 2, Toulouse, France \\ mathieu.guidere@univ-tlse2.fr
}

\begin{abstract}
RÉSUMÉ
Il y a quelques années, la constitution et l'exploitation des corpus publicitaires n'étaient pas une tâche aisée, mais la généralisation de l'internet comme outil de communication sociale, commerciale et institutionnelle a révolutionné ce champ d'études en rendant accessibles des sources d'information riches et variées. Les messages publicitaires sont désormais accessibles en ligne et en plusieurs langues, sur un même site web. Ils forment un corpus virtuel, multilingue et intersémiotique, offrant des textes parallèles et simultanés. Cette accessibilité autorise le traducteur à envisager de nouvelles approches et méthodes d'analyse. Tout d'abord, pour la constitution même des corpus d'étude, l'internet offre aujourd'hui la possibilité de réunir en un temps record des corpus multilingues et multimédias sans équivalent. Ensuite, pour leur exploitation, la comparaison des éléments constitutifs de ces corpus offre de nouvelles méthodes de recherche qui permettent d'envisager le traducteur comme un véritable communicateur. Enfin, en ce qui concerne la conceptualisation, une approche interdisciplinaire de ces corpus ouvre des perspectives inédites en matière d'études traductologiques et communicationnelles. Nous aborderons ces différents niveaux d'analyse en partant d'études de cas réalisées à une décennie d'intervalle à partir de corpus publicitaires multilingues. Notre objectif est d'expliquer l'évolution qui s'est opérée ces dernières années dans le domaine de la traduction publicitaire et de montrer l'intérêt d'une approche diachronique des corpus pour la traductologie.
\end{abstract}

\section{ABSTRACT}

A few years ago, dealing with advertisement corpus was not an easy task, but the generalization of the Internet as a tool of social and commercial communication has revolutionized this field of study by making available rich and varied sources of information. Ads and commercials are now available online and in several languages on the same website. They form a virtual corpus, which is multilingual and intersemiotic, offering simultaneous and parallel texts. This accessibility makes possible new approaches and methods of analysis for the translator. First, simply in forming the study corpus, the Internet now offers the opportunity to gather in record time unrivaled multimedia and multilingual corpus. Then, at the processing level, the comparison of corpus components offers new methods enabling the translator to be seen as a true communicator. Finally, in terms of conceptualization, an interdisciplinary approach to the corpus opens up new perspectives in translation studies. We discuss these different levels of analysis, starting from case studies conducted a decade earlier on the multilingual advertisement corpus. Our goal is to explain the evolution that has occurred in recent years in the field of advertising translation and the importance of a diachronic approach of corpora to translation studies.

\section{MOTS-CLÉS/KEYWORDS}

corpus, publicité, multilinguisme, sémiotique, cognition

corpora, advertisement, multilingualism, semiotics, cognition 


\section{Contexte}

En quelques années, la plupart des instituts de formation en langues sont passés de la traduction à la communication multilingue. Certes, l'essor des sciences de l'information et de la communication y est pour beaucoup, mais cela n'aurait pas été possible si la dimension multilingue n'était pas implicitement comprise dans le concept même de traduction. Désormais, traduire revient, en quelque sorte, à communiquer en plusieurs langues et le traducteur fait donc office de communicateur multilingue.

Ce changement de perspective reflète une évolution dans la théorie de la traduction et dans la pratique professionnelle. Avec l'autonomisation accrue de la discipline, la dimension linguistique est passée au second plan, comme un préalable évident, cédant le pas devant les préoccupations culturelles et communicationnelles. De plus, après avoir été longtemps cantonné dans la partie strictement langagière, le traducteur accède progressivement à de nouvelles fonctions et assume des responsabilités qui débordent largement le cadre de la traduction à proprement parler. Ainsi, par exemple, il participe à l'adaptation de campagnes publicitaires, intervient au sein des entreprises pour préparer les communications en langue étrangère, prend en charge la localisation des sites web en plusieurs langues, conseille les institutions et les organisations internationales sur la meilleure communication à adopter à l'égard de tel ou tel public cible, etc.

Dès 1997, Hatim et Mason avaient annoncé cette tendance en publiant Translator as Communicator. Dans ce livre, les auteurs appellent à une unification de la discipline - et des traducteurs - autour de leur point commun, à savoir la communication. Qu'ils soient traducteurs littéraires ou audiovisuels, ou encore interprètes ou médiateurs, ils sont tous des communicateurs. Ils précisent: «la traduction est envisagée comme un acte de communication qui tente de relayer un autre acte de communication pardelà les barrières linguistiques et culturelles» (Hatim et Mason 1997: 15).

À travers une série d'études de cas, les auteurs illustrent leurs propos en prenant un exemple de chaque domaine d'application, mais leur "modèle d'analyse» ne s'appuie pas sur un corpus de textes précis et rigoureux. Ils piochent leurs exemples au gré de leur besoin dans divers types de textes, en omettant même le plus communicatif des types textuels, à savoir le texte publicitaire.

Nous allons tenter de démontrer dans les pages qui suivent qu'une étude rigoureuse de la traduction comme communication interlangues ne peut ignorer les possibilités ni les avantages qu'offrent désormais les corpus publicitaires dans tous les domaines.

\section{Corpus}

Il est révolu le temps où le chercheur en traductologie devait guetter la parution, dans plusieurs magazines, de la même publicité, et où il devait mener une recherche documentaire à partir de la presse internationale. La constitution du corpus s'étalait alors sur plusieurs années avec une activité de veille soutenue sur les principaux mensuels et hebdomadaires dans les langues qui intéressaient sa recherche. En une décennie à peine, avec l'expansion et la généralisation de l'internet, le champ des études ayant recours aux corpus a connu une véritable révolution.

Aussi, le corpus publicitaire dont il est question dans le présent article est intersémiotique et multilingue, c'est-à-dire qu'il est accessible d'emblée en plusieurs langues et regroupe des éléments issus de plusieurs systèmes de signes (texte, son, image). Il 
s'agit, de surcroît, d'un corpus parallèle, qui présente le même message dans plusieurs langues, ce qui autorise un alignement du texte et de l'image en vis-à-vis (voir les figures 1 et 3).

Le multilinguisme est le trait le plus intéressant de ce corpus, mais les sites web déclinés en plusieurs langues offrent une telle variété de présentation qu’il est difficile de dresser une typologie fiable et stable des corpus qui en sont issus. Il est clair, cependant, que le corpus multilingue est devenu une réalité tangible qui se caractérise par le facteur temps. En effet, les diverses versions d'un même site sont mises en ligne de façon simultanée ou synchronisée.

Ces versions multiples d'une même communication forment le multitexte publicitaire. En théorie, la mise en place d'une version supplémentaire correspond à la volonté de suivre l'évolution du marché, en optimisant la communication à l'international. Dans la pratique de l'adaptation, cela se traduit généralement par de légères modifications dans la mise en forme du message qui accompagne l'image sur la publicité en ligne.

En conséquence, le corpus multilingue renvoie aux diverses mises en forme d'une même publicité accessible en ligne, c'est-à-dire aux différentes versions étrangères d'un même message promotionnel, quel que soit son degré d'adaptation au niveau écrit, sonore ou visuel. Dans la pratique, il existe peu de différences entre les versions que l'on peut explorer sur l'internet et celles qui sont publiées sur support papier, dans les magazines par exemple. Il s'agit foncièrement des mêmes arguments de promotion et des mêmes éléments iconiques, mais ceux-ci sont présentés selon une combinaison et un agencement légèrement différents.

Le multitexte publicitaire désigne la pluralité des textes diffusés pour une même publicité en plusieurs langues. Sur le plan langagier, le message n'est pas seulement traduit en un certain nombre d'idiomes, il est aussi décliné en plusieurs versions. Que ce soit au niveau national (par ex., la Suisse, le Canada) ou international (l'Europe, les États-Unis), il est possible de trouver différentes mises en forme d'un même message, sur un même site web. De ce point de vue, le multitexte renvoie également à la diversité des formulations et des aménagements que peut subir un même message au sein d'une même langue ou encore entre deux traditions culturelles d'une même langue. Les indices de l'identité, comme ceux de l'altérité, sont perceptibles tant au niveau de l'expression que de la mise en forme du message.

Enfin, sur le plan communicationnel, le multitexte désigne le résultat de différentes formes d'adaptation: réécriture, transposition ou encore simple transcodage. La pluralité des versions est ainsi le reflet d'un ensemble de normes et de contraintes formelles qui font la spécificité de la communication multilingue. La pluralité des versions n'est possible que s'il existe des normes techniques et rédactionnelles strictes. L'identité plurielle du message est le résultat d'une codification, aussi draconienne que fonctionnelle, des modalités de transfert interlangues. Cela signifie que la communication ne se réfère pas systématiquement à une langue source et à une culture d'origine; dans la pratique, elle s'effectue par le biais de compromis qui, dès le stade de création, intègrent l'idée de l'adaptation à plusieurs langues et cultures. De la sorte, il existe bien un message original, mais il est à envisager comme un Urtext (texte premier). La mise en évidence des différentes versions permet de retrouver l'esprit de la création originale, et leur comparaison incite à réfléchir sur les modes de communication interculturelle. 


\section{Méthode}

Si l'on admet que le corpus publicitaire multilingue reflète une forme de communication adaptée dans son contenu et dans ses modalités, on se rend compte qu'il participe à la continuité spatiale et temporelle des stratégies globales de communication. S'exerçant sur le langage, le travail du traducteur consiste à veiller attentivement à la stabilité du contenu tout en adaptant la forme du message. Les modalités d'adaptation linguistique et iconographique font l'objet d'une attention particulière, mais leur traitement peut poser des difficultés majeures en raison du caractère fondamentalement interdisciplinaire de la recherche.

Dans Publicité et traduction (Guidère 2000), l'approche choisie est «sémiotraductologique». Mais afin de mesurer le chemin parcouru depuis, il convient de préciser ici le cadre dans lequel cette étude avait été élaborée, ainsi que l'orientation théorique qui a présidé à ces recherches. En effet, les deux points essentiels et novateurs dans ladite étude concernent la nature du corpus étudié et la méthodologie de traitement des données.

\subsection{La constitution $d u$ corpus électronique}

Lorsque notre recherche a été entreprise dans les années 1990, la publicité internationale n'avait pas l'ampleur qu'on lui connaît aujourd'hui, mais l'on percevait déjà une montée en puissance des études fondées sur des corpus publicitaires choisis plus ou moins en fonction de leur disponibilité.

De plus, l'internet n'avait pas encore connu l'expansion et le succès que l'on observe aujourd'hui, ce qui signifie que les recherches en traductologie étaient encore centrées sur les problématiques traditionnelles du domaine: sens, équivalence, fidélité, liberté, adaptation, etc. Certes, l'École supérieure d'interprètes et de traducteurs de Paris (l'ESIT avec D. Seleskovitch et M. Lederer) avait apporté un regard nouveau sur le processus de traduction et établi le lien entre la traductologie et les sciences cognitives, mais les questions posées n'avaient que peu de rapport avec le traitement des corpus de textes spécialisés tels que les textes publicitaires.

Cela est d'autant plus vrai que la nouveauté et le renouveau des études traductologiques devaient venir de l'introduction massive des corpus de textes bilingues dans l'étude des faits de traduction. Peu d'études avaient été menées jusque-là sur des corpus parallèles ou comparables, et aucune ne portait sur un corpus publicitaire multilingue concernant les mêmes campagnes dans plusieurs langues. La double innovation de Publicité et traduction (2000) a été d'une part, d'introduire les problématiques liées aux corpus multilingues au sein de la traductologie; et d'autre part, d'explorer un domaine professionnel peu étudié jusque-là par les spécialistes: la traduction publicitaire.

L'étude concerne prioritairement les modes d'adaptation de la communication commerciale aux langues et cultures d'accueil. Pour mener à bien une telle recherche, il fallait un corpus valide et pertinent non seulement pour la constitution de l'objet même de l'étude mais également pour en montrer les enjeux et les limites.

Pour satisfaire aux exigences de scientificité des corpus, il fallait que les documents choisis répondent à des critères précis. Tout d'abord, le critère d'exhaustivité qui consistait à réunir toutes les publicités françaises du domaine, qui ont été traduites, adaptées 
et diffusées dans d'autres pays, en particulier dans les pays de langue arabe. Pour cela, il fallait faire une recherche longue et fastidieuse auprès des agences de publicité et des principaux diffuseurs de messages promotionnels à l'international afin de s'informer sur le lieu et la date de diffusion, les commanditaires, les coûts, les publics cibles, etc.

Ensuite, le critère d'unité et d'homogénéité qui exigeait de choisir un domaine précis de promotion publicitaire et une durée bien définie pour son étude. Le choix dans ce domaine a été aisé en raison de la prédominance constatée d'emblée dans le corpus des annonces publicitaires françaises en faveur des parfums et des produits de beauté. Cet état de fait s'explique d'ailleurs parfaitement par la position de leadership mondial qu'occupent les multinationales françaises dans ce secteur d'activité (L'Oréal, Garnier, Yves Rocher, Givenchy, Yves Saint-Laurent, Chanel, Dior, etc.). L'unité du domaine était donc assurée: ce sera celui des produits de beauté (parfums et cosmétiques).

Enfin, le critère de représentativité qui exigeait de sélectionner de façon représentative un ensemble d'annonces publicitaires issues des secteurs sus-indiqués. Mais il y avait également la représentativité temporelle et territoriale qui a été résolue au cours des années 1990 par le choix de n'étudier que les publicités diffusées dans des supports internationaux (revues internationales, journaux et magazines) où se trouvait l'essentiel des messages promotionnels traduits et adaptés à destination des publics étrangers.

La constitution d'un corpus qui satisfasse à l'ensemble de ces critères est un processus long, coûteux et difficile. Mais nous avons pu réunir, en faisant appel aux centres d'internationalisation des multinationales et aux principales agences de communication, un corpus publicitaire qui reste unique en son genre et inégalé à ce jour. En effet, le corpus final de l'étude a consisté en un millier de publicités françaises avec leurs équivalents en anglais, arabe et espagnol. Nous avons fait figurer de nombreux exemples de ce corpus multilingue dans l’ouvrage déjà cité (Guidère 2000).

\subsection{Le traitement du corpus électronique}

Cet immense corpus pour l'époque posait des problèmes redoutables sur tous les plans, aussi bien d'un point de vue théorique que pratique. La nouveauté de l'étude et l'originalité du domaine accentuaient cette difficulté, car il fallait, en même temps, procéder à l'analyse des données et créer le cadre d'une approche sémio-traductologique inédite, étant donné que ce genre de problématique mixte n'avait pas été véritablement abordé auparavant. Nous allons essayer de préciser ces deux points.

Le cadre théorique d'analyse du corpus textuel fut d'abord sémiolinguistique. Nous avons procédé d'emblée à la saisie et la classification de tous les textes publicitaires que nous avions à notre disposition, constituant par là même l'un des premiers et des plus importants corpus publicitaires électroniques.

Il existe au sein de la majorité des publicités trois niveaux textuels que nous avons veillé à transcrire fidèlement dans l'ensemble des langues répertoriées. Le premier niveau est celui de l'appellation (ou nom de marque) qui apparaît différemment selon les langues et qui est parfois transféré tel quel, d'autres fois translittéré de manière approximative en langue étrangère. Cette première distinction a donné lieu à un répertoire de noms propres et de noms communs qui posaient le problème traductologique 
du transfert des anthroponymes (transcription en caractères latins, adaptation culturelle, variation phonétique, etc.).

Le deuxième niveau textuel est celui du slogan, généralement constitué lui-même d'une "phrase d'accroche» (head-line) et d'une "phrase d'assise» (base-line). C'est la partie la plus importante de l'annonce publicitaire car elle a pour fonction d'" accrocher» le récepteur et de l'induire à mémoriser le nom de la marque ou le message porté par la publicité. Là encore, la classification a donné lieu à un corpus de phraséologies utiles pour la suite de l'étude. La problématique posée à ce niveau est celle de l'inscription de la praxis langagière au sein des structures phrastiques et de sa possible transposition d'une langue à l'autre: quel segment pour quel effet? Quelle traduction pour garantir le transfert de l'effet d'une langue à l'autre?

Le troisième niveau textuel est celui de la partie basse de l'annonce, que les spécialistes de la communication appellent «rédactionnel» et qui désigne le texte informatif ou argumentatif qui accompagne l'annonce publicitaire. Ce texte consiste généralement en un ou deux paragraphes qui détaillent les qualités et les avantages du produit, soit de façon explicite, soit de façon allusive. L'objectif d'un tel rédactionnel est bien évidemment la persuasion du récepteur par des procédés qui relèvent d'une rhétorique de l'action et de la manipulation que nous avons tenté d'expliquer à travers de nombreux exemples. Ainsi, nous avons réuni dans un même fichier tous les rédactionnels de nos annonces publicitaires afin de les soumettre à une étude formelle et thématique rigoureuse.

Mais parallèlement à l'identification et à la classification des niveaux signifiants du texte, nous avons mené une analyse approfondie de l'iconographie publicitaire. En effet, très rares sont les annonces du corpus qui ne sont pas accompagnées d'une image actualisant ou renforçant le sens textuel. L'un des premiers questionnements de ces images concerne leur fonction au sein de l'économie générale de l'annonce: à quoi servent-elles (fonction communicative)? et pourquoi sont-elles ainsi disposées (structure communicative)?

Pour répondre à ces questions, il a fallu procéder d'abord à une analyse sémiotique de l'ensemble des composantes de l'iconographie publicitaire. Ainsi, nous avons établi une typologie des images promotionnelles en fonction de trois éléments constitutifs du contenu thématique : l'objet publicitaire (parfum, crème, etc.), le personnage publicitaire (humain, animal, etc.), et le cadre publicitaire (naturel, urbain, etc.). Pour chacun de ces constituants, nous avons élaboré une typologie des signes constitutifs et des fonctions au sein du message.

Pour réaliser une telle étude, il a fallu recourir aux méthodes éprouvées de la sémiotique, qu'elle soit plastique ou picturale. La distinction peircienne entre «signe», «symbole» et «icône» nous a permis de mieux préciser les relations de sens existant entre les divers éléments de l'iconographie (Gorlée 1993). Mais il a fallu parfois étudier les techniques de composition et de prise de vue photographique pour comprendre le sens esthétique ou la finalité pratique des images analysées.

L'approche de ces éléments sémiotiques de manière comparative a donné lieu à une interrogation sur les fondements de la communication interculturelle au sein de la publicité internationale, sur ses méthodes et sur ses limites. Afin de répondre à ces interrogations cruciales pour la contextualisation des messages publicitaires, nous avons dû établir un répertoire des signes iconiques observables dans le corpus, avec un concordancier des adaptations imagières selon la publicité et le produit. 
Nous avons pu ainsi faire la démonstration de l'importance quantitative et qualitative de la charge culturelle et émotionnelle dans le transfert des éléments constitutifs de l'iconographie publicitaire. Mais au-delà, nous avons interrogé l'idéologie et les valeurs latentes décelées derrière les adaptations traductionnelles et les retouches d'image effectuées par les spécialistes de la communication au sein des agences publicitaires ou dans des officines de traduction et de localisation (Guidère 2004).

Le corpus iconographique qui a servi de point de départ à une telle étude se composait d'une base de données contenant des signes interconnectés au niveau des annonces mais dissociés au sein du corpus pour servir à l'établissement de comparaisons et de tendances générales dans l'exploitation de l'image publicitaire. L'une des applications les plus originales de ce type de travail consiste à définir l'image de marque d'un produit ou d'une entreprise en faisant une étude comparée des signes constitutifs des images publicitaires qui lui sont associées au cours d'une période donnée.

\section{Une méthodologie actualisée}

Ainsi, l'innovation portait à la fois sur l'objet d'étude (les publicités adaptées sémiotiquement et culturellement) et sur la nature des données étudiées (textes et images mis en parallèle). Mais avec la généralisation de l'internet, la révolution est venue de la quantité des messages publicitaires accessibles au chercheur par un simple clic. L'on se trouve, en effet, aujourd'hui face à un volume considérable de données exploitables dans la plupart des langues internationales (anglais, français, espagnol, arabe). Aussi est-il important de mettre à jour les approches et les méthodes de traitement de ces données dans le cadre de la traductologie.

Nous allons exposer brièvement cette nouvelle manière de traiter les corpus publicitaires multilingues, car nous voudrions insister sur le fait que cette méthodologie enrichit les études traductologiques en ayant recours aux corpus. Le message publicitaire, dans sa diversité linguistique et culturelle, doit être étudié à partir de ses différentes manifestations et des divers contextes de diffusion.

\subsection{Le traitement des données linguistiques}

Pour constituer un corpus publicitaire multilingue, il suffit aujourd'hui de visiter les sites web des entreprises multinationales et de naviguer entre les différentes versions accessibles en ligne. Ainsi, des compagnies aériennes comme Air France, Lufthansa ou British Airways, ou encore de grandes entreprises comme L'Oréal, Ford ou Nike, affichent, sur leur page d'accueil, des liens vers divers pays et langues d'accès. Aussi la constitution du corpus consiste-t-elle à mettre en parallèle les pages correspondantes dans différentes langues.

Une fois constitué et organisé, le corpus est soumis à une étude typologique menée selon une méthodologie spécialement adaptée au discours publicitaire et visant à en faire ressortir les spécificités et les éléments saillants. Ainsi, la méthodologie d'analyse adoptée pour le texte monolingue (français) est de nature sémiostylistique (étude des traits formels et rhétoriques du langage publicitaire). En revanche, pour l'analyse de la traduction (la version anglaise, arabe, espagnole), la méthode adoptée est celle de la sémiolinguistique (sur le plan microstructural) et de la linguistique textuelle (sur le plan macrostructural). 
Sur le plan technique, le corpus textuel peut être soumis à un traitement informatique selon une méthodologie de type lexicométrique. Ce traitement consiste avant tout à recenser, de façon automatisée, l'ensemble des catégories grammaticales présentes dans les textes du corpus, l'objectif étant de dégager des dominantes et des variantes lexicales entre systèmes linguistiques, concernant, par exemple, la qualification des produits et des services promus par la publicité dans chaque langue. Mais une telle étude serait déconnectée du sens véhiculé par l'image qui accompagne généralement le texte.

Il en est de même des termes techniques et scientifiques pour lesquels il est possible d'envisager une extraction automatique. Mais cela nécessite une conceptualisation suffisante en amont pour le domaine étudié (p. ex. les cosmétiques), qui permette d'approfondir cet aspect de la recherche. De même, il est possible d'en donner une représentation socioterminologique axée non pas sur la structure des termes observés mais sur leur diffusion en fonction de la cible visée par le produit: cosmétiques pour femmes, produits anti-âge, produits amincissants, produits de beauté, parfums pour hommes, produits pour sportifs, etc. À cet égard, la non-fermeture de la classe des publicités à étudier permet d'envisager une réactualisation régulière du corpus et une systématisation des résultats de l'étude. Mais là encore, elle ne peut se faire sans une réflexion sur la relation entre le texte et l'image.

Ainsi, pour le corpus écrit, nous avons pu élaborer une première classification et une première mise en correspondance des séquences sémantiques, en particulier pour les slogans publicitaires en lien avec les images. L'objectif d'une telle classification est de déterminer les représentations communes aux différentes langues étudiées (FR / EN / ES / AR). Ces représentations sont définies en tenant compte des phénomènes d'adaptation qui touchent les éléments iconographiques.

\subsection{Le traitement des données iconographiques}

Il fut un temps où l'étude d'un corpus visuel était difficile et coûteuse en raison de la rareté et de la complexité des outils de gestion de l'image. Mais avec les nouvelles possibilités offertes par les outils informatiques et infographiques, il est désormais possible d'envisager toutes sortes de traitements des images à des fins de recherche.

Tout d'abord, la numérisation intégrale du corpus est devenue la règle; elle permet d'avoir une vue d'ensemble de la totalité des images du corpus multilingue sous forme de diaporamas. Elle permet également d'envisager un classement du corpus par catégorie de produit et par type d'image. Ainsi pour ce qui est de notre corpus de référence, nous avons réuni, sous format électronique, toutes les images publicitaires présentant un modèle féminin, en fonction de l'angle de prise de vue, de la posture du personnage et de l'axe de communication adopté. Nous avons fait de même pour les différents objets et cadres publicitaires (cadre naturel, urbain, familial, etc.) en disposant en parallèle les images correspondant à chaque langue.

Il est possible également de procéder au "découpage» des images du corpus en dissociant, grâce à un logiciel de traitement spécifique, les différents éléments constitutifs de chaque iconographie et en les classant par type sémantique (animé, humain, concret, abstrait, etc.), par catégorie sémiotique (icône, symbole, indice) ou encore par procédé rhétorique de composition (métonymie, métaphore, personnification, etc.). 
À noter que les outils de reconnaissance graphique automatique sont désormais capables de traiter les images qui leur sont soumises afin de les étiqueter (tag) automatiquement. Ainsi, le logiciel Riya permet d'identifier les visages sur des photos et la société Google propose un outil de recherche visuelle (Image Labeler) qui permet aux internautes d'étiqueter les images qui leur sont proposées, l'objectif étant d'entraîner les futurs programmes de reconnaissance automatique pour la recherche d'images sur l'internet.

L'analyse automatique des images provenant de documents consiste à extraire et à reconnaître les informations présentes dans ces derniers sous forme numérisée. Les outils informatiques existants sont généralement inspirés des approches cognitives de la perception humaine, notamment pour la reconnaissance des formes. Ces outils traitent les images selon des indices perceptifs de type: contour, orientation, courbure, couleur, texture, etc. Mais ils peuvent également extraire automatiquement des indices visuels liés à des principes psycho-perceptifs de repérage et de catégorisation de l'information. L'intérêt principal de ces outils est qu'ils permettent de traiter des corpus de très grande taille, pour ne retenir que l'information saillante et pertinente.

Ces approches perceptives et cognitives offrent une voie nouvelle dans l'exploitation des corpus intersémiotiques tels que les corpus publicitaires. Elles autorisent toute une gamme de manipulations sur les images originales et sur les images adaptées dans la culture cible, ce qui permet de valider les hypothèses de sens ou d'efficience relatives à tel ou tel élément de l'ensemble. Ainsi, pour comprendre la valeur culturelle attachée à la forme d'un flacon de parfum par rapport à son appellation, il est possible de remplacer celui-ci par un autre en l'insérant directement dans l'image puis en interrogeant les récepteurs sur sa valeur et sur la relation nouvellement instituée entre les deux signes publicitaires: la forme et le nom; la couleur et la forme; le nom et la couleur, etc.

Ce type de traitement des données iconographiques bénéficie désormais des progrès importants des techniques infographiques et des méthodes d'analyse automatiques. L'objectif de ce genre de manipulations est de répondre à des questions telles que: que se passe-t-il lorsqu'on remplace tel énoncé par tel autre en gardant la même image d'une langue à l'autre? En changeant la couleur de fond? En modifiant la disposition du texte? En substituant les symboles?, etc.

Répondre à ce type d'interrogations à partir d'une investigation empirique est le préalable à la constitution d'une base de données iconographique riche et variée du point de vue des indications sémiotiques et culturelles relatives aux modalités d'adaptation du texte et de l'image: suppression, substitution, modification ou simple ajout d'éléments particuliers susceptibles d'influer sur le récepteur.

Dans le cadre de nos recherches, le résultat final montre clairement l'intérêt d'une approche cognitive et traductive des signes iconiques au sein des corpus publicitaires multilingues. Le problème posé est celui de la cohérence et de l'efficience des réseaux de signification iconographique dans le domaine publicitaire. On a beau croire que les créatifs de la publicité suivent leur imagination, il n'en reste pas moins que celleci révèle, à l'examen des productions effectives, des récurrences évidentes pour un même type de communication et des tendances indéniables dans l'adaptation aux marchés visés.

La combinaison de ces deux types d'approche, sémiolinguistique pour le texte et sémiocognitive pour les images, permet une étude globale de la signification publi- 
citaire par delà la diversité des éléments qui la composent. De ce point de vue, une telle recherche permet certes de mettre en évidence des aspects jusque-là considérés comme mineurs dans le langage publicitaire, mais elle montre également la spécificité irréductible de la traduction publicitaire, ni tout à fait technique, ni tout à fait littéraire.

\section{Pour une traductologie cognitive fondée sur l'usage de corpus}

Par traductologie cognitive, il faut comprendre l'étude de la traduction centrée sur la connaissance des moyens et des processus d'acquisition des compétences traductionnelles, c'est-à-dire l'ensemble des mécanismes par lesquels le traducteur acquiert de l'information à partir du texte source, la traite et l'exploite pour produire le texte cible. Dans cette perspective, la traductologie cognitive est amenée à s'intéresser aux grandes fonctions de l'esprit lorsqu'il traduit un document complexe et aux compétences liées au langage, au raisonnement, à la décision, etc.

L'un des modèles qui illustre cette prise en compte de la cognition dans le processus de traduction est celui du cube traductologique (Guidère 2009). Il est fondé sur l'analyse des conceptions (C), des perceptions (P) et des intentions (I), présentes dans le texte source (TS) et dans le texte cible (TC).

- Les conceptions renvoient aux concepts, c'est-à-dire aux idées générales présentes dans le texte. Elles se retrouvent généralement au niveau des noms propres et des noms communs du texte original et traduit.

- Les perceptions renvoient aux percepts, c'est-à-dire aux informations sensorielles que notre esprit traduit en émotions. Elles se retrouvent en général au niveau des images, des adjectifs et des adverbes des deux textes.

- Enfin, les intentions renvoient aux objectifs, c'est-à-dire aux projections et aux finalités du message ou du discours. Elles se retrouvent en général au niveau des verbes de sentiment et de l'implicite discursif de chaque texte.

Dans ce modèle, l'analyse se focalise sur le processus de transformation de la triade C-P-I du texte source en C-P-I du texte cible, en montrant les avantages et les limites des différents choix de traduction. Afin d'illustrer la pertinence de ce modèle pour une étude cognitive des corpus de textes publicitaires, nous proposons ci-après un exemple d'analyse sémiotraductologique en prenant comme exemple la publicité L'Oréal pour les crèmes de beauté disponible sur la Toile (voir figures 1, 2 et 3).

Pour ce faire, il faut d'abord identifier, dans chacune des publicités, les aspects qui méritent un commentaire à chaque niveau du texte, c'est-à-dire les mots et leurs traductions dont le sens connoté est différent en anglais et en français (conceptions), les images, dont la charge émotionnelle ou culturelle est différente dans les deux langues (perceptions); enfin, les formulations, dont la visée communicationnelle peut varier au moment de la traduction (intentions).

\subsection{Niveau des conceptions: le message linguistique}

Dans la publicité de RevitaLift ${ }^{1}$ (figure $1^{2}$ ), le message linguistique est centré sur le concept de jeunesse: le produit est censé préserver la jeunesse, c'est son argument de vente. Le concept s'oppose dans la phrase d'accroche à la notion de dureté suivant une logique simple: le produit permet de rendre le visage «moins dur » et, par conséquent, de paraître «plus jeune». 


\section{Figure 1}

\section{Publicité de L’Oréal: RevitaLift}

A - Version française: France

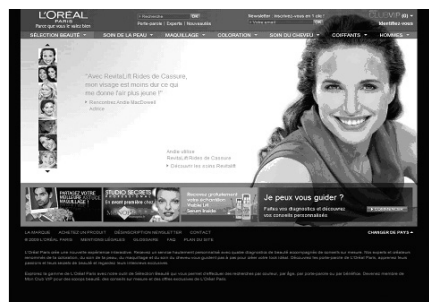

"Avec RevitaLift Rides de Cassure, mon visage est moins dur, ce qui me donne l'air plus jeune!"

- Rencontrez Andie McDowell Actrice

Andie utilise

RevitaLift Rides de Cassure

- Découvrir les soins Revitalift
B - Version anglaise: Grande-Bretagne

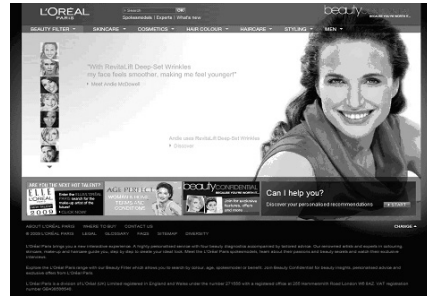

"With RevitaLift Deep-Set Wrinkles my face feels smoother, making me feel younger!"

- Meet Andie McDowell

Andie uses RevitaLift Deep-Set Wrinkles

- Discover

Pour comprendre le sens de jeunesse dans le message français, il faut faire une déduction logique, par antithèse. L'air jeune est ainsi lié à l'idée de douceur, mot qui n'est jamais prononcé, mais que l'on peut déduire par opposition à dur et à Cassure (composante du nom du produit). Ainsi, le produit donne l'impression de jeunesse parce qu'il rend les traits du visage plus doux. Mais le message dit explicitement que le produit «donne l'air plus jeune», il ne procure pas véritablement la jeunesse, ce n'est pas un élixir de jouvence. L'implicite discursif est axé sur le paraître jeune et non sur l'être jeune, il promeut l'apparence et non l'essence. Pour acheter le produit, la consommatrice doit adhérer à plusieurs postulats en relation avec le concept central du message: 1) la jeunesse est dans l'apparence; 2) la jeunesse est dans le visage; 3) la jeunesse du visage est dans sa douceur. Si la consommatrice adhère - consciemment ou inconsciemment - à ces idées, alors elle sera convaincue de l'intérêt et des mérites du produit promotionné. Ainsi, à partir d'une analyse de la logique discursive, il est possible de préciser le sens du concept central autour duquel est construit le message publicitaire en français.

La comparaison avec la version anglaise de la même publicité montre des différences notables sur le fond, bien que la formulation soit comparable (structure com-

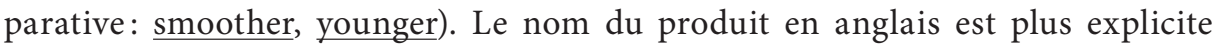
concernant son champ d'application ("Wrinkles») et la logique discursive mise en œuvre affiche une spécificité indéniable: le message n'est pas centré sur l'apparence («l'air jeune») mais sur la sensation (« feel younger»). Le modèle (Andie McDowell) dit explicitement: «my face feels...», «making me feel...», littéralement «mon visage se sent...», «je me sens...».

Lors de la traduction, nous passons donc d'un message centré sur l'objet (le visage, l'apparence) à un message centré sur le sujet (le locuteur et ses sensations). Dans un cas, le produit modifie l'apparence de la chose, dans l'autre, il modifie le ressenti de l'humain. Sans toucher à l'image publicitaire, la traduction transforme un message centré sur le paraître (le visible) en un discours centré sur l'être (le ressenti). De la 
sorte, la perception d'une image, pourtant identique, par les réceptrices dans chaque langue devient différente: en français, elle est l'illustration de l'efficacité concrète du produit (le visage est visiblement plus doux et donc plus jeune), tandis qu'en anglais, elle est l'expression de l'effet du produit sur l'utilisateur (le large sourire reflète la sensation procurée par le produit). Indéniablement, la comparaison des deux versions révèle un changement de perception par lequel le texte donne sens à l'image.

\subsection{Niveau des perceptions: le message iconique}

Dans la publicité sur Vive Pro (figure 2), le message iconique est centré sur le modèle (Penélope Cruz). Comme l'image de ce modèle est différente entre les deux versions, française et américaine, le regard est immédiatement attiré par cette différence, et cette focalisation oriente l'interprétation du message linguistique qui l'accompagne. On lit le texte en référence à l'image et non l'inverse. Or, que «dit» cette image précisément?

FIGURE 2

Publicité de L'Oréal: Vive Pro

A - Version française: France

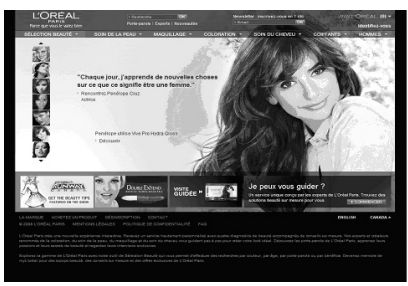

«Chaque jour, j’apprends de nouvelles choses sur ce que signifie être une femme.»

- Rencontrez Penélope Cruz Actrice

Penélope utilise Vive Pro Hydra Gloss

- Découvrir
B - Version anglaise: Grande-Bretagne

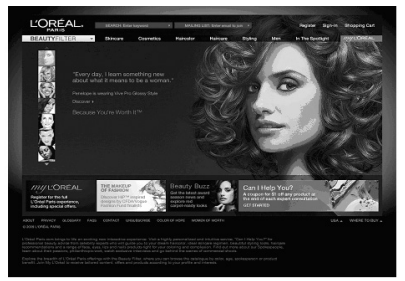

"Every day, I learn something new about what it means to be a woman."

Penelope is wearing Vive Pro Glossy Style Discover

Because You're Worth It ${ }^{\mathrm{TM}}$

Tout d'abord, l'arrière-plan de l'image est différent: neutre en français, marqué en anglais. Associé au teint travaillé du modèle, cet arrière-plan signale implicitement la cible potentielle du message publicitaire: femmes blanches en France $v s$ femmes de couleur aux États-Unis.

La coiffure du modèle tend à renforcer ce ciblage en associant l'esthétique afroaméricaine aux origines latines du modèle, aisément décodables à travers le nom (Penélope Cruz) dont l'accent disparaît d'ailleurs sur la version américaine (Penelope). Les cheveux longs et lissés du modèle en français sont un symbole de modernité et de liberté chez la femme.

La posture du modèle n'a pas la même signification sur les deux versions publicitaires. En français, le regard, dirigé directement vers la lectrice et associé au sourire, renforce la perception d'une femme libre qui «n'a pas froid aux yeux». En synergologie, la tête penchée du modèle indique la douceur du « regard social» et l'empathie par rapport à l'interlocutrice (Turchet 2000: 80-83). 
À l'inverse, sur la version américaine, l'angle de prise de vue (de profil) permet de mettre en scène un «regard d'échange» ayant une dimension «mystérieuse» (Turchet 2000: 137-144) en raison de l'œil droit partiellement voilé par les cheveux du modèle. La femme garde ici son mystère et ses mots doivent être lus à la lumière de cette part de mystère. La forme des lèvres dessine une expression différente du sourire affiché par le modèle sur la version française. En anglais, les lèvres indiquent un état de parole en cours ou sur le point de se dire: «la clôture de la bouche par rapport à la bouche ouverte marque toute la différence entre écouter et entendre» (Turchet 2000: 170). Entre désir et censure, les lèvres du personnage publicitaire renvoient à la phrase inscrite en vis-à-vis: "Chaque jour, j’apprends de nouvelles choses sur ce que signifie être une femme».

En raison des différences notables dans le visuel publicitaire, la perception de cet «être femme / to be a woman» dont il est question dans le rédactionnel s'avère également différente. C'est l'iconographie qui détermine cette perception de «l'être femme». La présence du slogan de la marque sur la version américaine uniquement («Because You're Worth It») contribue à cette différenciation. Mais le sens précis de l'image dans chacune des versions relève d'une relation subtile entre l'explicite et l'implicite dans la mise en forme du message publicitaire.

\subsection{Niveau des intentions: l'explicite et l'implicite}

Que signifie le message publicitaire? Quelle est l'intention du locuteur? Dans la publicité Excell $10^{4}$ (figure 3), les bras croisés du modèle (Eva Longoria) évoquent visuellement l'assurance, mais le message linguistique est centré sur le temps et la perfection, deux idées qui incitent plutôt à relativiser cette posture assurée.

FIGURE 3

Publicité de L'Oréal: Excell 10

A - Version française: France

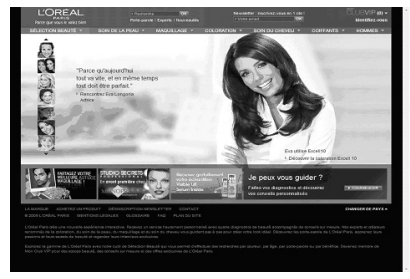

"Parce qu'aujourd'hui tout va vite, et en même temps tout doit être parfait."

- Rencontrez Eva Longoria Actrice

Eva utilise Excell 10

- Découvrir la coloration Excell 10
B - Version anglaise: Grande-Bretagne

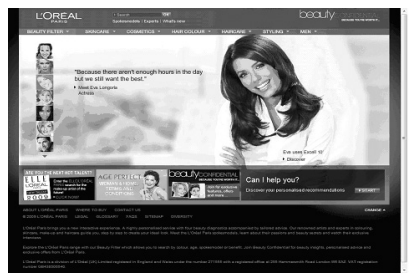

"Because there aren't enough hours in the day but we still want the best."

- Meet Eva Longoria Actress

Eva uses Excell 10’ [sic]

- Discover

Que dit le texte en français et pourquoi le dit-il sous cette forme? Telle est la question centrale de l'intentionnalité. «Parce qu'aujourd'hui tout va vite, et en même temps tout doit être parfait», déclare la mannequin, sûre d'elle, en français. Ce qu'elle dit explicitement, c'est que l'époque a changé et que ce changement est marqué par 
une accélération du rythme et des exigences de la vie contemporaine. Mais le «Parce que» initial indique la troncature du texte et la présence d'un implicite discursif. Ce qui est dit implicitement, c'est que les humains n'ont pas changé dans leur désir de perfection et qu'il faut leur apporter une aide pour réaliser ce désir: c'est la raison d'être du produit (Excell 10), dont le nom même évoque l'excellence comme médiation vers la perfection tant recherchée. Du point de vue intentionnel, nous sommes dans l'empathie: consciente des exigences de la vie moderne et en même temps des limites des humains, la marque (L'Oréal) comprend et aide ses clientes à travers son message et grâce à ses produits. L'essence du message est maternelle, comme l'indique l'attitude générale du modèle (sourire, regard, bras croisés) : «Rassurez-vous, je suis là, la vie est belle.»

En anglais, le message est plus pratique, plus concret, puisqu' «il n'y a pas assez d'heures dans la journée» ("there aren't enough hours in the day»). Le modèle ne vise pas la perfection mais «cherche le meilleur» («want the best»), tout simplement. Ce que dit la marque implicitement est qu'elle offre «le meilleur» à ses clientes. S'il n'y avait pas la mention «coloration» sur la version française, on ne saurait même pas de quel produit il s'agit précisément, tant le message vise à généraliser. L'intention latente du discours est de conférer une qualité générale à l'ensemble des produits de la marque. La fonction d'ancrage du texte nous guide dans la lecture pour donner un sens à l'image sur le registre de la positivation; s'il n'y avait pas de texte, on pourrait percevoir l'image de façon aléatoire.

Ainsi, à travers un choix de mots et de signes visuels, la publicité oriente le message dans la langue source et dans la langue cible de façon différente. C'est le sens même de la communication orientée (Guidère 2009). Ainsi conçue, une traductologie cognitive basée sur corpus permettrait de concilier le produit et le processus dans l'étude de la traduction publicitaire.

\section{Conclusion}

L'un des apports majeurs de la recherche sur corpus multilingue a consisté en la constitution de la traduction publicitaire comme objet d'étude spécifique et fondamentalement interdisciplinaire. Jusqu'ici, peu d'études d'ensemble ont été réalisées sur un vaste corpus électronique, mais les enquêtes que nous avons menées ces dernières années montrent le caractère inopérant des cadres et modèles d'analyse traditionnellement appliqués aux autres types de traduction (littéraire, technique, scientifique).

En effet, le travail sur corpus a permis de mettre en évidence l'essor indéniable de la communication multilingue sur la Toile mondiale, ainsi que de nouvelles formes d'intervention du traducteur dans le monde virtuel. Ces évolutions requièrent de la part des chercheurs en traductologie l'intégration de la dimension cognitive de la communication à partir d'une exploitation rigoureuse des corpus intersémiotiques.

\section{NOTES}

1. Publicité de L’Oréal (Revitalift). Consultée le 15 juin 2010, <http://www.loreal-paris.fr/soin-de-lapeau/soin-du-visage/revitalift.aspx\#/home>.

2. La légende de chacune des publicités a été retranscrite en respectant la ponctuation et la typographie utilisées dans l'original (notamment les guillemets). 
3. Publicité de L'Oréal (Vive Pro). Consultée le 15 juin 2010, <http://www.lorealparisusa.com/_us/_en/ default.aspx\#/?page=top\{userdata//d+d//|diagnostic|main :brandpage :vivepro|media:_blank|nav| overlay:_blank\}>.

4. Publicité de L'Oréal (Excell 10). Consultée le 15 juin 2010, <http://www.loreal-paris.fr/coloration/ couleur/excell-10.aspx\#/discover/>.

\section{RÉFÉRENCES}

GorLéE, Dinda L. (1993): Semiotics and the Problem of Translation: With Special Reference to the Semiotics of Charles S. Peirce. Amsterdam: Akademisch Proefschrift.

Guidère, Mathieu (2000): Publicité et traduction. Paris: L'Harmattan.

Guidère, Mathieu (2003): La création lexicale en arabe moderne. Turjumân. 12(1):107-121.

Guidère, Mathieu (2004): De l'adaptation à la localisation publicitaire. In: James ARCHIBALD, dir. La localisation: problématique de la formation. Montréal: Linguatech, 69-96.

Guidère, Mathieu (2009): De la traduction publicitaire à la communication multilingue. Meta. 54(3):417-430.

Hatim, Basil and Mason, Ian (1997): The Translator as Communicator. London and New York: Longman.

Turchet, Philippe (2000): La synergologie. Québec: Éditions de l'Homme. 